\title{
Digital Control For KEK-PS MA Loaded RF System
}

\author{
M. Yoshii, E. Ezura, Y. Hashimoto, C. Ohmori, \\ Y. Mori, A. Takagi, KEK, Tsukuba 305-0801, Japan \\ M. Yamamoto, JAERI, Tokai 319-1195, Japan \\ A. Schnase, COSY, Forschungszentrum Juelich GmbH, Juelich, Germany
}

\begin{abstract}
The magnetic alloy loaded radio-frequency system has been installed in the KEK $12 \mathrm{GeV}$ synchrotron. This system is a high field gradient radio-frequency system and is designed to operate not only as a fundamental harmonic system to increase the insufficient matching voltage, supplied by 4 conventional ferrite cavities but also as a second radiofrequency system to alleviate the space charge effects during beam injection. Digital signal processing is the technique to process the continuous machine or beam signals and to couple with a direct digital synthesis, which is the way to generate a phase controlled precise frequency signal. Both techniques are indispensable for the low-level radio-frequency controls of a complex system. Developments of DDS based radio-frequency signal generator and digital phase detector and related ADC modules have been started for this new MA radio-frequency system. The details of $R \& D$ equipments are going to be reported.
\end{abstract}

\section{INTRODUCTION}

Since the successful system installation, the KEKPS magnetic alloy loaded radio-frequency system has been intermittently operated during the KEK-PS physics runs. And during these test operations, the parameters as a second harmonic system have been optimized. This system is a high electric field gradient system, which has been developed for one of the $R \& D$ issues of the JAERI-KEK-Joint (JKJ) project [2]. Two of the $150 \mathrm{~kW}$ tetrode tubes consist in the final stage of radio-frequency amplifier, which is employed to supply the power into the cavity. The inverter DC plate supply using the insulated gate block transistors (IGBT) has also been developed to realize small output voltage ripples and to solve the problem lies on a protection circuit such as a claw-bar.

Digital control is the most attractive subject in employing the low-level radio-frequency (LLRF). Since the MA cavity is a passive load, a so-called, "direct frequency drive" is possible. The DDS-NCO, high speed ADC and a digital phase detector have been developed for this system.

\section{PS MA RF SYSTEM}

\subsection{General}

The KEK-PS magnetic alloy loaded radio-frequency cavity is designed to cover the frequencies of both the fundamental $(\mathrm{h}=9)$ and second radio frequency $(\mathrm{h}=18)$. The cut core technique realizes that the magnetic material loaded radio-frequency system operates at different resonant conditions [1]. Two frequency bands can be switched by changing the air gap distance between the pair of split toroidal cores inside.

The KEK PS MA system has been tuned at the frequency of $12 \mathrm{MHz}$. This is the mode to use the system as a second harmonic system.

TABLE 1.Typical performances of KEK-PS MA system

$\begin{array}{ll}\text { Peak accelerating voltage: } & 30 \mathrm{kV} \\ \text { Rf-frequency (MHz): } & 12.04 \mathrm{MHz} \\ \text { Harmonic number: } & 18 \\ \text { Number of gaps: } & 2 \\ \text { Shunt impedance per gap: } & 1000 \Omega \\ \text { Quality factor: } & 10 \\ \text { Air gap distance: } & 60 \mathrm{~mm} \\ \text { Peak power dissipation: } & 220 \mathrm{~kW} \\ \text { Number of MA cores: } & 8 \\ \text { Length of cavity: } & 95 \mathrm{~cm}\end{array}$

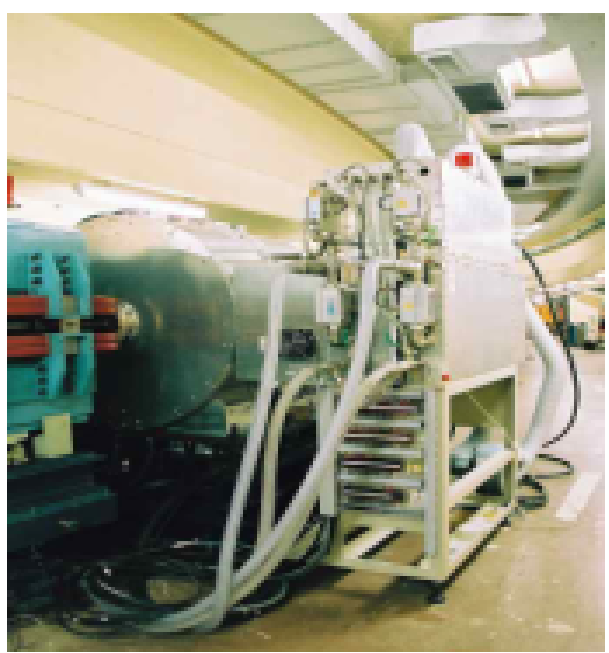

Figure1. The KEK-PS MA RF System 


\subsection{Plate DC Power Supply}

The inverter DC power supply has many advantages regarding output ripple, stability and controllability, compared to unstabilized transformer/rectifier power supplies. The inverter/converter configuration decouples the output from the fluctuation of primary AC line of $6600 \mathrm{~V}$. Because of the high chopping frequency for IGBT modules, the ripple frequency can be entirely separated from the synchrotron frequency. Also, the output voltage sag becomes tolerable under the high repetitive operation in case of a rapid cycle synchrotron.

The plate power supply for MA radio-frequency system consists of the 12-phase Thyristor rectifier, DC inductance (DCL) and the output IGBT modules. The IGBT modules are connected in parallel. Each of them shares $66 \mathrm{~kW}$ among 400kW maximum rating. Nominal output DC voltage is $13 \mathrm{kV}$ and the output current varies from a few Amps quiescent current to maximum 30A according to the operating pattern. (Figure 2)

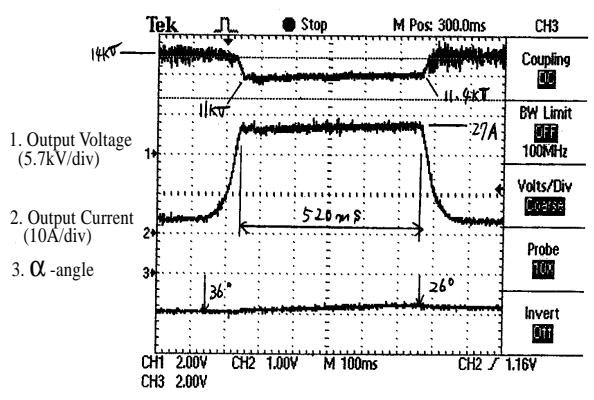

Figure.2 Typical waveforms of the plate DC power supply; (a) an output voltage $(5.7 \mathrm{kV} / \mathrm{div})$, (b) an output current (10A/div) and (c) an alpha phase angle for the input Thyristors are shown.

\section{CONTROLS}

A significant feature of a magnetic alloy core lies in its stability against high magnetic field and temperature variation. As the MA loaded cavity is a passive load system, so-called, "direct frequency drive" is possible.

The present low-level radio-frequency control for the KEK-PS is based on the voltage control oscillator (VCO). The digital control system is the best solution to generate an exact time variable rf-signal in the place of an analog indirect system (VCO).

\subsection{Direct Digital Synthesis (DDS)}

The Qualcomm Q2240I-3S1 based DDS numerical oscillator has been designed and developed for utilizing to the KEK $12 \mathrm{GeV}$-PS radio-frequency systems instead of the present VCO LLRF control. With the same IC chips and ideas, a VME board for a different purpose has been designed at COSY, Juelich Germany, which synthesizes band-limited noise for slow beam extraction [3].

The DDS board composes of Qualcomm DDS chip, 16-bit digital adder for phase modulation, two coordinate transformers (TMC2330) and a 14-bit DAC (AD9754). The DDS board runs with an $80 \mathrm{MHz}$ clock. Since the coordinate transformer runs below a $50 \mathrm{MHz}$ clock, however, the phase signal out of DDS is split to run under a $40 \mathrm{MHz}$ clock and fed into the two coordinate transformers set in parallel. And each transformed signal is interleaved again before the DAC's input. The $80 \mathrm{MHz}$ oscillator is a TXCO crystal and the temperature dependence is less than a few ppm. The stability of DDS output directly reflects the clock characteristic. The output frequency error has been achieved to be less than $\pm 5 \mathrm{~Hz}$ at a $12 \mathrm{MHz}$ output frequency.

The DDS module provides a 16-bit phase control input and a 16-bit amplitude control input. These input signals are going to be controlled by a commercial DSP (TMS320C6211) module.

\subsection{Fast Digital Phase Monitor (FDPM)}

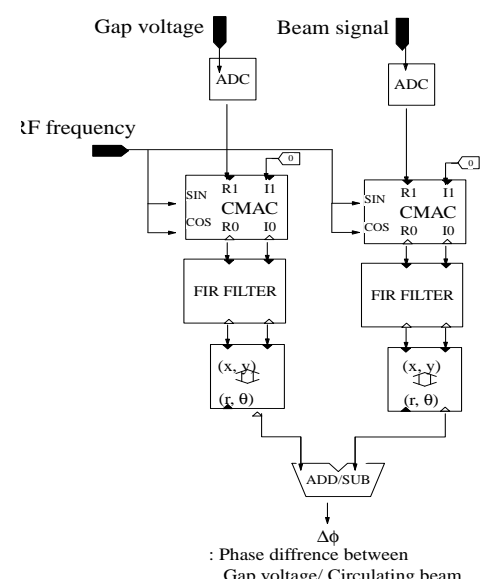

Figure 3. Block Diagram of the Fast Digital Phase Monitor (FDPM)

In order to suppress the amplitude of dipole oscillation due to the beam phase errors, a $100 \mathrm{kHz}$ 
bandwidth is necessary in $\Delta \phi$ feedback. A fast digital phase monitor (FDPM) has been designed to detect the time variable phase differences between the cavity and the circulating beam. And, this 16-bit output word of phase is connected to the DSP module. The FDPM module consists of two 12-bit analog-to-digital converters (AD9432), which sample the data signals of both the gap voltage and circulating beam, 16-bit multiplier/accumulators (CMAC, HSP45116AVC), digital low pass filters and the coordinate transformers (TMC2330A). The arithmetic formulae in a 16-bit multiplier/accumulators,

$$
\begin{aligned}
\text { Ro } & =\mathrm{R} 1 \cos \theta-\mathrm{I} 1 \sin \theta, \\
\mathrm{Io} & =\mathrm{I} 1 \cos \theta+\mathrm{R} 1 \sin \theta,
\end{aligned}
$$

where (Ro, Io) and (R1, I1) are complex output and input, respectively. So, when I1 $=0$ and R1 is the cavity voltage or beam signal, one gets each of the phase angles in a polar coordinate $(\mathrm{R}, \theta)$.

Digital low pass filtering is necessary to eliminate high frequency components of CMAC output. The sampling frequency of this FDPM module was selected $40 \mathrm{MHz}$, and the cut-off frequency was selected $800 \mathrm{kHz}$. The FIR filter is designed as a digital lowpass filter. The high-speed programmable logic device (ALTERA APLEX20K) has been used for this FIR filter.

\section{SUMMARY}

The magnetic alloy loaded radio-frequency system has been installed in the KEK 12 GeV-PS. This is a high field gradient cavity and the $30 \mathrm{kV}$ of accelerating voltage is achieved at the frequency of 12 MHz. This cavity is operated as the second harmonic system now. The IGBT inverter DC power supply is designed and successfully operated. For the accomplishment of the digital RF control, the DDS NCO module and the fast digital phase monitor have been developed. The DDS module runs with a very precise accuracy and reliability. But, the FDPM module needs more parameter optimization for its FIR digital filter. The MA system will be completely combined with the present fundamental radiofrequency system, after the low-level radio-frequency system has been updated to the digital system.

\section{ACKNOWLEDGEMENT}

The authors would like to thank Professor I. Yamane for generous support and valuable discussions.

\section{REFERENCE}

[1] M.Yoshii el al., "MA RF Cavity for the KEK 12GeV PS", EPAC2000 (2000) p.984-986.

[2] C.Ohmori el al., "JKJ Synchrotron RF System", KEK-Proceedings 2000-22, ICANS-XV TSUKUBA, Nov.2000, p.252

[3] Discussions about "noise generation" at the $255^{\text {th }}$ International WE-Heraeus-Seminar, "Beam Cooling and Related Topics, Forschungszentrum Juelich, May 13 - 18, 2001 